\title{
Transferring Quantum Information through the Quantum Channel using Synchronous Multiplexing for Multiple users
}

\author{
H.R.Sumathi ${ }^{1}$ and C.Vidya Raj $^{2}$ \\ ${ }^{1}$ Dept of Information Science and Engineering, JSS Academy of Technical Education \\ Bengaluru 560060, Karnataka,India. \\ ${ }^{2}$ Principal, NIE Institute of Technology Mysuru 570018, Karnataka, India. \\ Affiliated to Visvesvaraya Technological University, Belagaum 590014, Karnataka, \\ India.
}

\begin{abstract}
:
Transmission of information in the form of qubits much faster than the speed of light is the important aspects of quantum information theory. Quantum information processing exploits the quantum nature of information that needs to be stored, encoded, transmit, receive and decode the information in the form of qubits. Bosonic channels appear to be very attractive for the physical implementation of quantum communication. This paper does the study of quantum channels and how best it can be implemented with the existing infrastructure that is the classical communication. Multiple access to the quantum network is the requirement where multiple users want to transmit their quantum information simultaneously without interfering with each others.
\end{abstract}

\section{KEYWORDS:}

Qubits, Quantum memory, Bosonic channel, Optical fibre, Multiplexing

\section{INTRODUCTION:}

An attractive physical system for representing a quantum bit is the optical photon. Photons are charge less particles and do not interact with each other or even with most matter. They can be guided along long distances with low loss in optical fibres, delayed efficiently using phase shifters and combined easily using beam splitters[1].A single photon can carry at most one bit of classical information. Quantum information theory is fundamentally richer than classical information theory, because quantum mechanics includes so many more elementary classes of static and dynamic resources. Quantum information theory contains many more facts other than described here, including the study of quantum operation, quantum error correcting codes, Quantum data compression. The unique properties of quantum states are it is impossible to copy because of no cloning theorem also they cannot be perfectly distinguished this is quantified by the Holevo bound[2].The Holevo bound on the accessible information is not an easy theorem, but like many good things in quantum information theory, it follows easily once the strong subadditivity of Von Neumann entropy is established.

$$
\mathrm{I}(\mathrm{X} ; \mathrm{Y}) \leq \chi(\varepsilon)
$$




\section{COHERENT QUANTUM INFORMATION AND CAPACITY:}

The coherent information $\mathrm{I}_{\mathrm{e}}$ is given by,

$$
\mathrm{I}_{\mathrm{e}}=\mathrm{S}\left(\rho^{\mathrm{Q}}\right)-\mathrm{S}_{\mathrm{e}}
$$

The coherent information has many properties that suggest it as the proper measure of the quantum information conveyed from sender to receiver by the channel. For example, $\mathrm{I}_{\mathrm{e}}$ can never be increased by quantum data processing performed by receiver on the channel output, and perfect quantum error correction of the channel output is possible if and only if no coherent information is lost in the channel[3]. The coherent information seems to be related to the capacity of a quantum channel to convey quantum states with high fidelity[4].

\section{ENCODING OF QUANTUM INFORMATION:}

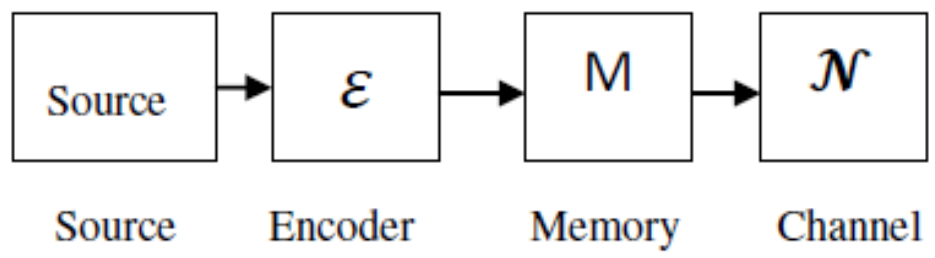

Fig. 1

The source is quantum information, it is encoded into quantum states. Before transmitting it is stored in quantum memory. When transmitting qubits, the information is encoded into nonorthogonal superposed or entangled quantum states chosen from the ensemble $\left\{\rho_{\mathrm{k}}\right\}$ according to a given probability $\mathrm{P}_{\mathrm{k}}$. If the states $\left\{\rho_{\mathrm{k}}\right\}$ are pure and mutually orthogonal, it reduces to classical. The quantum channel represents a completely positive, trace preserving map[2]. A class of channels known as bosonic channels,which are a quantum mechanical model for free space or fiber optic communication.

\section{OPTICAL NETWORKS}

These are called synchronous networks because the timing is tightly controlled. Synchronous networks exhibit very accurate timing.

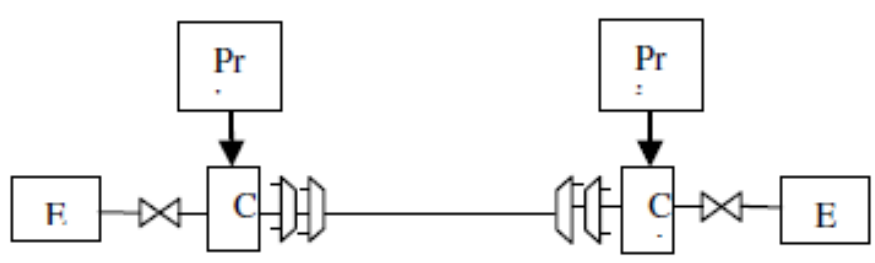

Fig. 2

Pr $\rightarrow$ Primary reference source(PRS)

E $\rightarrow$ End Terminal

C $\rightarrow$ Cross connect

A single synchronous network uses one PRS, which is Known as the Master clock. Timing is derived first from the master clock [5]. 


\section{BOSONIC CHANNEL CAPACITY}

In quantum mechanics the state of a physical system together with the measurement that is made on that system determines the statistics of the outcome of that measurement. Thus, in seeking the classical information capacity of a bosonic channel, the optimization must be allowed for both the transmitted quantum states and the receivers quantum measurement. In particular it is not appropriate to immediately restrict consideration to coherent state transmitters and coherent detection or direct detection receivers. What is needed for deducing the fundamental limits on optical communication which is the analog of Shannons noisy channel coding theorem-that applies to transmission of classical information over a noisy quantum channel viz the HolevoSchumacher-Westmoreland(HSW) theorem[10].

Recent work on determining the channel capacity of optical channels is extended in several ways. Classical capacity is derived for a class of Gaussian Bosonic channels represent the quantum version of classical Gaussian-noise channels. The proof is strongly motivated by the standard technique of whitening Gaussian noise used in classical information theory. Minimum output entropy problems related to these channel capacity derivations are also studied. These single-user Bosonic capacity results are extended to a multi- user scenario by deriving capacity regions for single-mode and wideband coherent-state multiple access channels. An even larger capacity region is obtained when the transmitters use non-classical Gaussian states, and an outer bound on the ultimate capacity region is presented as well. The goal of quantum information theory is evaluating the information capacities of important communication channels. At present, exact capacity results are known for only a handful of channels. Here we consider the classical capacity $C$ of Bosonic channels with isotropic Gaussian noise. The study connects to a research line that began with the capacity derivation for the lossless Bosonic channel The Holevo-SchumacherWestmoreland(HSW) theorem quantifies explicitly the amount of classical information that can be transmitted through the noisy quantum channel $\mathcal{N}$ if the input contains product states(i.e., entanglement is not allowed). The information capacity of a noiseless channel is defined as the maximum number of bits that can be reliably sent per channel use[6]. In case of a classical channel it is given by the maximum of the Von Neumann entropy.

$$
\mathrm{S}(\rho)=-\operatorname{Tr}\left[\rho \log _{2} \rho\right]
$$

Over all the possible input states $\rho$ of the channel. In the interaction of an intense laser beam with a nonlinear medium, Therefore physically the non linear medium constitutes the channel and the photons of the laser beam carries the information. So the quantum states $\rho$ are the states of the photons which are bosons with zero rest mass. For a mass less bosonic field the associated Hilbert space is infinite dimensional and as a consequence of the infinite dimensional Hilbert space, the maximum entropy is infinite[7]. In a real situation a quantum channel having infinite capacity does not make sense and for all realistic scenarios a cutoff is introduced by constraining the energy required in the storage or in the transmission, eg. Requiring the entropy $\mathrm{S}(\rho)$ to be maximized only over those states having average energy $\mathrm{E}$ i.e $\mathrm{E}=\operatorname{Tr}[\rho \mathrm{H}]$ where $\mathrm{H}$ is the system Hamiltonian[7].

The constrained maximization of $\mathrm{S}(\rho)$ can be solved by standard variational methods which entail the solution of following equation

$$
\boldsymbol{\delta}\left\{\mathrm{S}(\rho)-\lambda_{1} / \ln 2 * \operatorname{Tr}[\mathrm{H} \rho]-\lambda_{2} / \ln 2 * \operatorname{Tr}[\rho]\right\}=0
$$

Where $\lambda_{1}$ and $\lambda_{2}$ are lagrange multipliers that take into account the energy constraint and the normalization constraint $\operatorname{Tr}[\rho]=1$, and the $\ln 2$ factor is introduced so that all subsequent 
calculations can be performed using natural logarithms. The above equation can be solved by the density matrix

$$
\rho=\exp (-\beta \mathrm{H}) / \mathrm{Z}(\beta),
$$

Where $\mathrm{Z}(\beta)=\operatorname{Tr}(\exp (-\beta \mathrm{H}))$ is the partition function of the system and is determined from the constraint, by solving

$$
\mathrm{E}=-\partial / \partial \beta * \ln (\mathrm{Z}(\beta))
$$

The corresponding capacity is thus given by

$\mathrm{C}=\mathrm{S}[\exp (-\beta \mathrm{H}) / \mathrm{Z}(\beta)]=(\beta \mathrm{E}+\ln \mathrm{Z}(\beta)) / \ln 2$.

For more realistic and physical systems one can't obtain exact analytic expression for C [8].

\section{MULTIPLE USERS}

When two or more users want to share the same Bosonic channel different multiple access schemes have to be chosen. Here in our paper we explain about the theoretical way to organise the photons in a linear form to form a frame with $\mathrm{m}$ qubits.If there are $\mathrm{n}$ users who want access the channel which as more capacity using round robin fashion each user can transmit their frames this is called Multiplexing. As it has to demultiplex at the receiver side proper synchronization is required. The existing infrastructure of fibre optic cables and standards like SONET/SDH and the optical transport network defined by ITU-T with Add/Drop multiplexing. As the capacity of optical systems increases, opportunities are created for network service providers to provide more capacity to the systems users. This approach allows the Network to operate synchronously while accepting asynchronous traffic. The ITU-T has published a layered model for optical networks, the general scheme is shown below. The model is designed with several layers and multiplexing operation are collectively called the optical transport hierarchy The functions of the layers are:[5].

Optical channel layer: Provides end to end optical channels between two optical nodes, supporting user payloads of different formats such as ATM, STM-n etc. Services include routing, monitoring, provisioning and backup and recovery features.

Optical multiplex section layer: Provides for the support of WDM signals and manages each signal as an optical channel. Services include wave length division multiplexing and multiplex section.

Optical transmission section layer: Provides the transmission of the physical optical signal, based on the specific type of fibre. Services include the correct signal generation and reception.

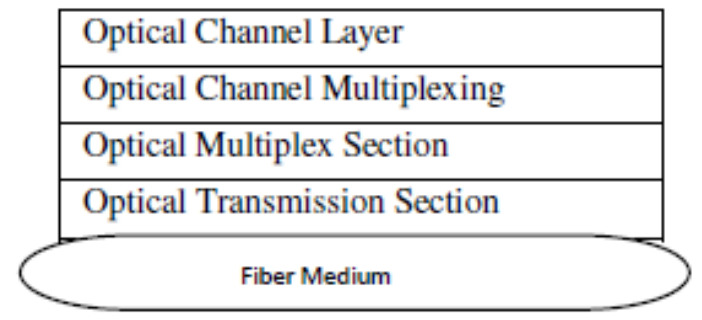

Fig. 3

The Fiber medium can be either wired or wireless optical communication. 


\section{CONCLUSION}

To know the capacity and to increase the transmission rate of a Quantum Bosonic channel is still a open problem. As per the IBM research group present classical communication itself provide much higher transmission rate than any quantum effects. A powerful aspect of an optical communication link is that many different wavelengths can be sent along a single fiber simultaneously in the 1300 to $1600 \mathrm{~nm}$ spectral band, this is wave length division multiplexing. Advanced dense and coarse WDM must be properly spaced to avoid inter channel interference.

Seeing the advantages of quantum information theory with no cloning theorem and Quantum Key Distribution $(\mathrm{QKD})$ along with quantum cryptography we can assume that quantum channels are much more secure than classical channel. Chinese have published that by 2016 they are launching a quantum satellite.

\section{REFERENCES}

[1] Michael A Nielsen \& Isaac L. Chuang "Quantum Computation and Quantum Information" Cambridge University Press First South Asian Print,(2002)

[2] Sandor Imre and Laszlo Gyongyosi “Advanced Quantum Communication” an engineering approach IEEE press Wiley publications,(2013).

[3] H. P. Yuen and M. Ozawa, Phys. Rev. Lett. 70, 363 (1993).

[4] A. S. Holevo, IEEE Trans. Inf. Theory 44, 269 (1998)

[5] Optical Networks, Uyless Black

[6] P. Hausladen, R. Jozsa, B. Schumacher, M.Westmoreland, and W. K.Wootters, Phys. Rev. A 54, 1869 (1996)

[7] V. Giovannetti, S. Lloyd and L. Maccone, "Capacity of nonlinear bosonic systems", Phs.Rev.A70(2004),012307

[8] Navneet K Sharma and Anirban Pathak, "Bosonic channel capacity of a Non linear dispersive optical fiber"'(2008)

[9] Jeffrey H. Shapiro, V. Giovannetti, S. Lloyd, L. Maccone, J. H. Shapiro, and B. J. Yen, "Capacity of Bosonic Communications" MIT, Research Laboratory of Electronics, Cambridge, USA.NEST-INFM and QUIT,Italy.

[10] Jeffrey H. Shapiro "Classical capacities of Bosonic Channels" 